typeset using JPSJ.sty $<$ ver.1.0b $>$

\title{
Enhancement of the Thermal Conductivity in gapped Quantum Spin Chains
}

\author{
Keiji Saito and Seiji Mryashita \\ Department of Applied Physics, Graduate School of Engineering University of Tokyo, Bunkyo-ku, Tokyo \\ $113-8656$
}

(Received November 8, 2018)

\begin{abstract}
We study mechanism of magnetic energy transport, motivated by recent measurements of the thermal conductivity in low dimensional quantum magnets. We point out a possible mechanism of enhancement of the thermal conductivity in gapped magnetic system, where the magnetic energy transport plays a crucial role. This mechanism gives an interpretation for the recent experiment of $\mathrm{CuGeO}_{3}$, where the thermal conductivity depends on the crystal direction.
\end{abstract}

KEYWORDS: Quantum Magnetism, Thermal Conductivity

\section{$\S 1$. Introduction}

The relationship of the thermal conductivity and magnetic state in various low dimensional quantum magnets has been attracted interests. experimental instrument to investigate the magnetic properties in materials. For the magnetic systems with large exchange coupling constants, the magnetic energy transport dominantly contributes to the thermal conductivity. Low-dimensional spin systems with many conserved quantities tend to possess a ballistic energy transport, which means an infinite thermal conductivity as studied in the theoretical side 0 , 8, These facts can give a possible mechanism of a large magnetic thermal conductivity. Solgubenko et al. observed such nondiffusive magnetic energy transport in $\mathrm{SrCuO}_{2}$ and $\mathrm{Sr}_{2} \mathrm{CuO}_{3}$ which are described by the quasi-one dimensional isotropic antiferromagnetic Heisenberg model. 1 These materials have very large exchange coupling constant $J \sim 2000 K$ so that the magnon runs through the system even at $100 \mathrm{~K}$. They found that the energy is transmitted by magnon at this temperature.

Unusual enhancement of conductivity is observed at low temperatures below a spin gap temperature in gapped spin system such as the two-dimensional dimer spin system $\mathrm{SrCu}_{2}\left(\mathrm{BO}_{3}\right)_{2}$, and the ladder system $(\mathrm{Sr}, \mathrm{Ca})_{14} \mathrm{Cu}_{24} \mathrm{O}_{41}$, 明, 目, This enhancement is attributed to the contribution of phononic energy transport. This phononic energy transport is quantitatively explained in view of

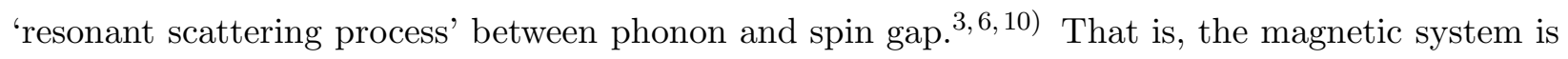


excited absorbing a phononic energy, and another phonon of the same energy is emitted deexciting the magnetic system at the same time.10) These experiments suggest that at high temperatures the magnetic energy transport dominantly convey the heat, while at low temperatures the magnetic gap suppresses the magnetic energy transport. Thus phononic energy transport become dominant. Therefore the energy carrier changes from spins to phonons around the spin gap temperature.

The spin-Peierls (SP) system is another gapped system where the energy gap is formed below a critical temperature $T_{\mathrm{SP}}$. $\mathrm{CuGeO}_{3}$ is a typical material which shows the SP transition. Ando et al. studied the thermal conductivity in the chain direction in $\mathrm{CuGeO}_{3}$ and found the enhancement of the conductivity below $T_{\mathrm{SP}} 11$ which is similar to that found in the two-dimensional dimer and


increase of the phononic transport due to the decrease of spin-phonon coupling interaction. The similar enhancement is also observed more clearly in $\mathrm{NaV}_{2} \mathrm{O}_{5}$ where the charge ordering transition occurs 12,13

Recently Hofmann et al. compared the properties of thermal conductivity of $\mathrm{SrCu}_{2}\left(\mathrm{BO}_{3}\right)_{2}$ and $\mathrm{CuGeO}_{3}$. 15 ) They investigated the thermal conductivity along different crystal axes, although the previous experiment in $\mathrm{CuGeO}_{3}$ was done only in the chain direction.11) Generally temperature dependence of phononic thermal conductivity does not depend on the crystal direction, whereas a magnetic one does on it. Thus by studying the crystal direction dependence of thermal conductivity, whether the enhancement of the thermal conductivity is caused by phonons or not is clarified. $\mathrm{SrCu}_{2}\left(\mathrm{BO}_{3}\right)_{2}$ is described by the Shastry-Sutherland model 4 ) which has the exact dimerized ground state and almost dispersionless triplet excitations where the magnetization is localized. Thus below the spin gap temperature, we expect that the phononic energy transport is dominant because of small magnetic contribution. Actually this prospect is supported by the observation that the thermal conductivity does not depend on the crystal direction. On the other hand, they found that $\mathrm{CuGeO}_{3}$ shows the direction dependence of thermal conductivity.

In this paper, motivated by these recent experiments, we theoretically investigate the role of magnetic transport when the system has the energy gap, and propose a mechanism of enhancement of the thermal conductivity in magnetic systems. We consider a bond-alternating antiferomagnetc Heisenberg chain, and investigate the thermal conductivity focusing on the dependence on the energy gap. By the quantum master equation approach, we study a spin system which is connected to two reservoirs of different temperatures. We found that the thermal conductivity is enhanced due to the presence of bond-alternation around the spin gap temperature. This observation gives an interpretation that a small bond-alternation yields an enhancement of specific heat but does not drastically change the mean free path. This interpretation is consistent with Hofmann's recent experiments.15)

This paper is organized as follows. In $\S 2$, we introduce the model, and the method to investigate 
the thermal conduction is explained in $§ 3$. Summary and discussions are given in $\S 4$.

\section{$\S 2 . \quad$ Model}

The system we shall consider is an alternate Heisenberg spin chain described by,

$$
\mathcal{H}_{s}=J \sum_{\ell=1}^{N-1}\left(1-(-1)^{\ell} \delta\right) \mathbf{S}_{\ell} \cdot \mathbf{S}_{\ell+1},
$$

where $J$ is an exchange interaction which is taken as a unity throughout this paper, and $\mathbf{S}_{\ell}$ is the $\ell$ th spin. The number of spins $N$ is taken to be even. A finite value of parameter $\delta$ causes a bond-alternation, which yields the dimerized ground state and a finite spin gap 17 We study the thermal conductivity for various values of $\delta$.

The thermal conductivity $\kappa(T)$ at the temperature $T$ is generally given by the Green-Kubo formula which reads as,

$$
\kappa(T)=\lim _{t \rightarrow \infty} \lim _{N \rightarrow \infty} \frac{1}{2 N T^{2}} \int_{0}^{t} d u\langle\{\hat{J}, \hat{J}(u)\}\rangle,
$$

where $\{.,$.$\} means the anti-commutation relation, and \langle\ldots\rangle$ is the equilibrium average at the temperature $T$. The operator $\hat{J}(u)$ is the total heat current operator at the time $u$ in the Heisenberg picture, i.e., $\hat{J}(u)=\exp (i \mathcal{H} u) \hat{J} \exp (-i \mathcal{H} u)$. The total current operator $\hat{J}$ for $(2.1)$ is calculated by the continuity equation of the energy as,

$$
\begin{aligned}
\hat{J} & =-i J \sum_{\ell=1}^{N-2}\left(1-\delta^{2}\right)\left[\mathbf{S}_{\ell} \cdot \mathbf{S}_{\ell+1}, \mathbf{S}_{\ell+1} \cdot \mathbf{S}_{\ell+2}\right] \\
& =-J\left(1-\delta^{2}\right) \sum_{\ell=1}^{N-2}\left(\mathbf{S}_{\ell} \times \mathbf{S}_{\ell+1}\right) \cdot \mathbf{S}_{\ell+2}
\end{aligned}
$$

where $[.,$.$] means the commutation relation. In the case of the isotropic Heisenberg model with the$ periodic boundary condition, i.e. $\delta=0$, the total current $(2.3)$ is a conserved quantity. Therefore the Green-Kubo formula (2.2) diverges, which means that magnons are not scattered and the mean free path of each magnon diverges. In real magnetic materials, the relaxation time becomes finite due to the other sources such as spin-phonon scattering and impurities, and so on.

In order to take these sources into account for the isotropic case $\delta=0$, more practical formulation of the thermal conductivity is used especially when the magnetic low excitation contributes to the energy transport at low temperatures, 1 , 18)

$$
\kappa(T)=v_{\mathrm{G}}^{2} \int d k \mathcal{D}(k) \tau(k) \frac{d}{d T}\left[\frac{\hbar \omega(k)}{e^{\beta \hbar \omega(k)}+1}\right],
$$

where $\omega(k)$ is the low energy dispersion which is taken to be the Des Cloizeaux-Pearson mode21) $\omega(k)=\frac{J \pi}{2} \sin k . \quad v_{\mathrm{G}}$ is the group velocity. $\mathcal{D}(k)$ and $\tau(k)$ are the density and the relaxation time of the mode $k$, respectively. Eq. (2.4) can be also written as $\kappa=\sum_{k} C_{k}(T) D_{k}(T)$ where $C_{k}(T)=\mathcal{D}(k) \frac{d}{d T}\left[\frac{\hbar \omega(k)}{e^{\beta \hbar \omega(k)}+1}\right]$ and $D_{k}(T)=v_{\mathrm{G}}^{2} \tau(k) . C_{k}(T)$ and $D_{k}(T)$ are interpreted as the specific 
heat and the diffusion constant of energy for the mode $k$, respectively. This is derived with the same spirit in the phononic transport discussed by the Peierls-Boltzmann equation.19) The relaxation time $\tau(k)$ is determined by various origins such as impurities and spin-phonon coupling, and so on. Practically, the relaxation rate is calculated by the Carraway's method,20) i.e., by the summation over relaxation rates originated from these origins. In this formula, the mean free path $l_{k}$ is defined using the relaxation time $\tau(k)$ as $l_{k}=v_{\mathrm{G}} \tau(k)$. In $\mathrm{CuGeO}_{3}$, above $T_{\mathrm{SP}}$ where the Hamiltonian is roughly regarded as the isotropic Heisenberg chain $\delta=0$, the mean free path is estimated as 500 lattice size 11] We should note that the mean free path is a dynamical property and is different from the spin correlation length in the equilibrium state.

In order to apply the formula (2.4) to the case of finite $\delta$, we must know the low energy dispersion with finite energy gap, although we do not know exact analytical expression for it. A finite energy gap causes the localization of the magnetic excitation which suppresses the energy diffusion, whereas energy gap enhances the heat capacity around spin gap temperature. For instance, when $\delta=1$, the system is completely separated into $N / 2$ local spin pairs, and the group velocity is 0 . Therefore this extreme case gives zero thermal conductivity due to vanishing mean free path. Thus it is not trivial whether the existence of energy gap enhances thermal conductivity or not. In this context, we will study the thermal conduction by a direct numerical method.

\section{$\S 3 . \quad$ Method and Results}

We investigate how the thermal conductivity behaves as a function of $\delta$. It is difficult to treat the Green-Kubo formula (2.2) numerically because it requires infinite $N$. Therefore the quantum master equation approach is more tractable in numerical simulations. We study the spin system which is directly connected to the reservoirs of different temperatures at the ends. We here consider a system of $N=6$ and $N=8$. Although this lattice sizes are small, we believe that the essential mechanism of magnetic energy transport is clarified. The time-evolution of the system is determined by the following master equation (e.g., see the reference 16 ) and references therein);

$$
\frac{\partial \rho(t)}{\partial t}=-i[\mathcal{H}, \rho(t)]-\lambda\left(\mathcal{L}_{\mathrm{L}} \rho(t)+\mathcal{L}_{\mathrm{R}} \rho(t)\right)
$$

where the first term in the right-hand side corresponds to the pure quantum dynamics of the system, and $\mathcal{L}_{\mathrm{L}}$ and $\mathcal{L}_{\mathrm{R}}$ express the contact with the thermal reservoirs of the inverse temperature $\beta_{\mathrm{L}}\left(=1 / T_{\mathrm{L}}\right)$ at the left end and $\beta_{\mathrm{R}}\left(=1 / T_{\mathrm{R}}\right)$ at the right end, respectively. The parameter $\lambda$ is the coupling strength. The dissipative term $\mathcal{L}_{\alpha}(\alpha=\mathrm{L}, \mathrm{R})$ is given by

$$
\mathcal{L}_{\alpha} \rho(t)=\left(\left[X_{\alpha}, R_{\alpha} \rho(t)\right]+\left[X_{\alpha}, R_{\alpha} \rho(t)\right]^{\dagger}\right),
$$

where $X_{\mathrm{L}}$ and $X_{\mathrm{R}}$ are the system's operators directly attached to the left and right reservoir, respectively. Here we take $X_{\mathrm{L}}=S_{1}^{z}$ and $X_{\mathrm{R}}=S_{N}^{z}$. The operator $R_{\alpha}$ is given by $\left\langle k\left|R_{\alpha}\right| m\right\rangle=\left(E_{k}-E_{m}\right)\left(e^{\beta_{\alpha}\left(E_{k}-E_{m}\right)}-1\right)^{-1}\left\langle k\left|X_{\alpha}\right| m\right\rangle$ in the representation diagonalizing the Hamil- 
tonian (2.1) as $\mathcal{H}|k\rangle=E_{k}|k\rangle$ and $\mathcal{H}|m\rangle=E_{m}|m\rangle$. If $\beta_{\mathrm{L}}=\beta_{\mathrm{R}}$, the master equation (3.1) gives the canonical distribution as the stationary solution, which is easily checked by substituting the canonical distribution for the density matrix $\rho(t)$. We numerically integrate the equation (3.1) and obtain the stationary density matrix $\rho_{\text {st }}$ starting from the initial density matrix of the canonical distribution at temperature $T_{\mathrm{R}}$. The simulation was carried out by the fourth order Runge-Kutta method with the time step 0.01 with $\lambda=0.01$.

We define the thermal conductivity by measuring the stationary energy current $\operatorname{Tr}\left(\rho_{\mathrm{st}} \hat{J}\right)$ setting

$$
T_{\mathrm{R}}=T_{\mathrm{L}}+\Delta T
$$

where $\Delta T$ is a temperature difference between two reservoirs, and is taken as $\Delta T=0.3$. Thereby the thermal conductivity $\kappa(T)$ is defined as

$$
\kappa\left(T=T_{\mathrm{L}}\right)=\frac{\operatorname{Tr}\left(\hat{J} \rho_{\mathrm{st}}\right)}{\Delta T} .
$$

We present the results of the thermal current $\kappa(T) \Delta T$ normalized by $N-2$, which is the number of terms in the summation (2.3) In Fig.1(a) and (b), the normalized thermal currents are shown for $N=6$ and $N=8$, respectively. In the inset, the data of the specific heat are also shown. The normalized thermal current show little quantitative difference between the cases of $N=6$ and $N=8$. In the figures, we obtained finite thermal conductivities even in the isotropic case, $\delta=0$. As explained in previous section, it must diverge in the Green-Kubo formula. These finite values of thermal conductivity are attributed to the finite size effect in the presence of the thermal reservoirs at ends of the system, and the conductivities must diverge in the limit of $N \rightarrow \infty$. Actually the conductivity for $N=8$ is larger than that for $N=6$. This size dependence is also observed for the cases of finite values of $\delta$.

In the isotropic case, $\delta=0$, the overall form of the thermal conductivity is similar to that obtained by Klümper and Sakai who exactly calculated the amplitude of zero frequency contribution in the Green-Kubo formula.22 That is, the thermal conductivity has one peak at about $T \sim 0.5$. This behavior is a common characteristic which does not change when the system size increases. At very low temperatures $\kappa(T)$ should be in proportional to $T$ due to the temperature dependence of specific heat, which is similar to the Casimir's theory in phononic transport.23) This low temperature property, however, cannot be checked for such finite sizes. We must note that even in the isotropic case $\delta=0$, the system has a finite energy gap due to the finite size effect. When $\delta$ becomes finite, the gap increases, e.g., in the case of $N=8, \Delta E=0.39269, \Delta E=0.74750,1.09041,1.41106,1.71290$, and 1.85807 for $\delta=0.0,0.2,0.4,0.6,0.8$, and 0.9 , respectively.

Small bond alternations enhance the thermal conductivity in spite that the alternation tends to separate the system into local spin pairs. This enhancement is observed for even $\delta=0.8$, and this feature is quite robust. In the case of very large $\delta$, the thermal conductivity is reduced due to the 
effect of separation of the system. The temperature of the peak of thermal conduction roughly corresponds to half of energy gap like the Shotky-type specific heat. Actually the overall behavior of thermal conductivity is similar to that of the specific heat. Thus from this observation, we conclude that the mean free path does not drastically changed by the bond-alternation. We expect that the unusual enhancement of the thermal conductivity in the spin-Peierls system is caused by magnetic energy transport when $T_{\mathrm{SP}}$ is the same order of $\Delta E$. That is, in the spin-Peierls system, $\kappa(T)$ follows the data of $\delta=0$ at the high temparture region, whereas below $T_{\mathrm{SP}}, \delta$ suddenly increases and $\kappa(T)$ is given by $\kappa(T, \delta \neq 0)$, which gives a sharp peak when $T_{\mathrm{SP}}$ is the same order of $\Delta E$. In $\mathrm{CuGeO}_{3}, T_{\mathrm{SP}}$ is the same order of $\Delta E$, i.e., $T_{\mathrm{SP}} \sim 14 K$ and $\Delta E \sim 20 K$.

\section{$\S 4 . \quad$ Discussion}

Magnetic energy transport is becoming a strong instrument to capture the magnetic properties of quantum magnets. In order to realize an accurate interpretation for obtained experimental data, theoretical arguments are necessary. We demonstrate how the thermal conductivity is enhanced due to the magnetic interaction, and proposed that besides the enhancement due to the increase of phononic energy transport, magnetic energy transport also causes an enhancement at a temperature of the order of $\Delta E$. This enhancement is directly related to that of the specific heat. This correspondence indicates that the mean free path does not drastically changed by a small bond-alternation. If the bond-alternation disturbs the dynamics of the system and the mean free path becomes much smaller than the isotropic chain, this correspondence between the thermal conductivity and specific heat is no more valid. The numerical simulation, however, indicates that there is some region where this mechanism of enhancement really exists. We hope that this effect by magnetic energy transport is also studied in experiment.

So far, it is difficult to control the heat flow in material. However making use of the magnetic energy transport, we may plan a new device that controls the energy flow. In such device, the energy flow could be drastically changed by controlling some parameters such as external magnetic fields. The creation of a energy gap provides a promising mechanism which enhances the energy flow. It is interesting to study mechanisms to control magnetic transport in more detail, which will be reported elsewhere.

\section{Acknowledgement}

The computer calculation was partially carried out at the computer center of the ISSP, which is gratefully acknowledged. The present work is supported by Grand-in-Aid for Scientific Research from Ministry of Education, Culture, Sports, Science, and Technology of Japan.

[1] A.V.Sologubenko, K. Gioannò, H. R. Ott, A. Vietkine, and A. Revcolevschi: Phys. Rev. B 62 (2000) R6108 ,Phys. Rev. B 64 (2001) 054412-1. 
[2] K. Kudo et al.: J. Phys. Soc. Jpn 70 (2001) 1448.

[3] M. Hofmann et al.: Phys. Rev. Lett. 87 (2001) 047202-1.

[4] K. Kudo et al.: J. Phys. Soc. Jpn 70 (2001) 437.

[5] A.V.Sologubenko, K. Gioannò, H. R. Ott, U. Ammerahl, A. Revcolevschi, D. F. Brewer, and A. L. Thomson: Physica B 284-288 (2000) 1595.

[6] A.V.Sologubenko, K. Gioannò, H. R. Ott, U. Ammerahl, and A. Revcolevschi: Phys. Rev. Lett. 84 (2000) 2714.

[7] K. Saito, S. Takesue, and S. Miyashita: Phys. Rev. E, 54 (1996) 2404.

[8] X. Zotos: Phys. Rev. Lett. 82 (1999) 1764.

[9] S. Fujimoto and N. Kawakami: J. Phys. A 31 (1998) 465.

[10] Neelmani and G. S. Verma: Phys. Rev. B 6 (1972) 3509.

[11] Y. Ando et al.: Phys. Rev. B 58 (1998) R2913, J. Takeya et al.: Physica B 284-288 (2000) 1645, J. Takeya et al.: Phys. Rev. B 61 (2000) 14700, J. Takeya et al.: Phys. Rev. B 62 (2000) R9260, J. Takeya et al.: Phys. Rev. B 63 (2001) 214407.

[12] A. N. Vasil'ev et al.: Phys. Rev. Lett. 81 (1998) 1949.

[13] V. V. Pryadun et al.: Physica B 259-261 (1999) 990.

[14] B. S. Shastry and B. Sutherland: Physica B 108 (1981) 1069, S. Miyahara and K. Ueda: Phys. Rev. Lett. 82 (1999) 3701.

[15] M. Hofmann et al.: Physica B 312-313C (2002) 597.

[16] K. Saito, S. Takesue, and S. Miyashita: Phys. Rev. E 61 (2000) 2397.

[17] R. G. Kepler: J. Chem. Phys. 39 (1963) 3528.

[18] L. D. Fadeev and L. A. Takhtajan: Phys. Lett. 85A (1981) 375.

[19] P. G. Klemens: Solid State Physics 7 (1958) 1, R. P. Tye ed.: Thermal conductivity, Academic Press 1969, R. Berman: Thermal Conduction in Solids, Clarendon Pres, Oxford, 1976

[20] J. Callaway: Phys. Rev. 113 (1959) 1046.

[21] J. des Cloizeaux and J. J. Pearson: Phys. Rev. 128 (1962) 2131.

[22] A. Klümper and K. Sakai: J. Phys. A 35 (2002) 2173.

[23] H. B. G. Casimir, Physica (Amsterdam) 5 (1938) 495. 

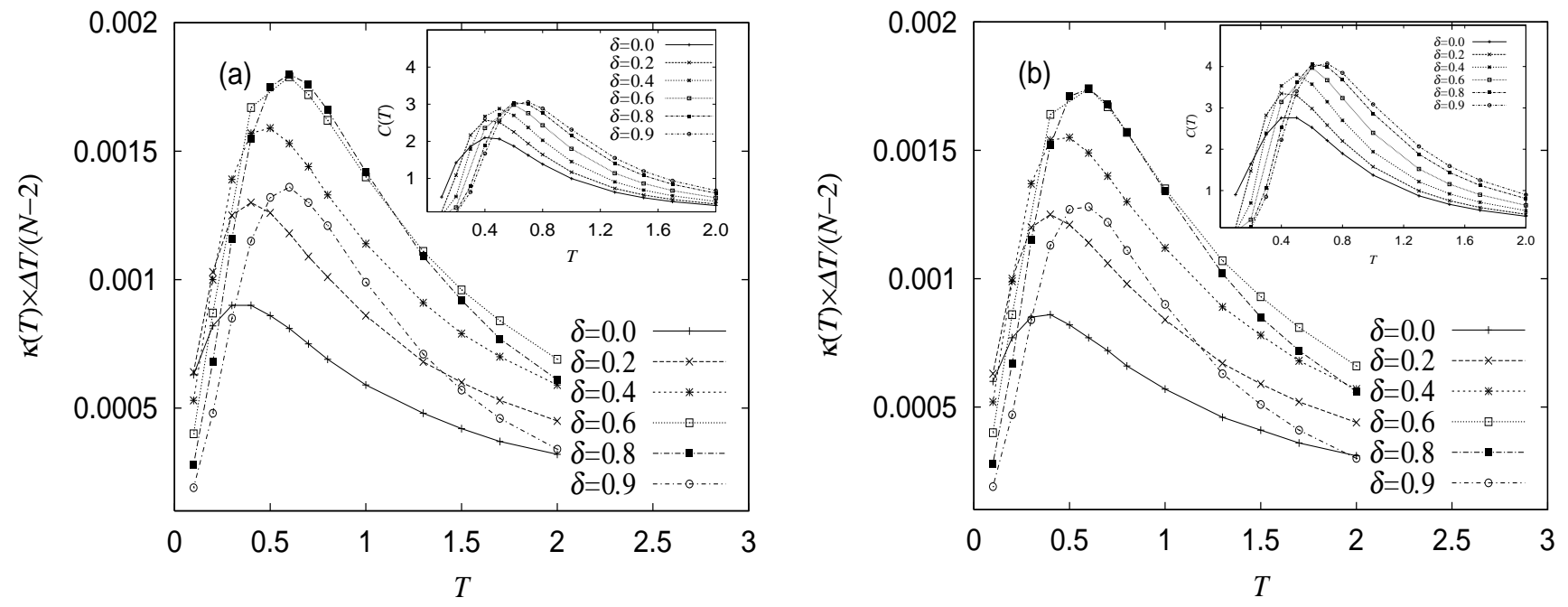

Fig. 1. The thermal conductivities for various $\delta$ with the system size, (a): $N=6$ and (b): $N=8$. The inset is the specific heat.

K. Saito and S. Miyashita 